\title{
Efficacy of Pseudomonas aeruginosa eradication regimens in bronchiectasis
}

\author{
To the Editor: \\ Patients with bronchiectasis and chronic infection with Pseudomonas aeruginosa have more frequent \\ pulmonary exacerbations and hospital admissions, and reduced quality of life and survival, than those who \\ are free of $P$. aeruginosa infection [1]. Guidelines published by the British Thoracic Society recommend \\ treatment to eradicate $P$. aeruginosa when first isolated in respiratory tract samples of people with \\ bronchiectasis [2]. However, the best regimen to achieve eradication and how to determine successful \\ eradication are yet unknown.
}

At the Northern Ireland Regional Respiratory Centre (Belfast, UK), the preferred eradication regimen is a combination of 6-week oral ciprofloxacin and 3-month nebulised colistimethate sodium [3]. However, this regimen is varied according to patient experience (e.g. drug allergy and/or intolerance), clinician judgement and the antimicrobial susceptibility profile of the $P$. aeruginosa isolate. Therefore, the aims of this study were to determine if the eradication regimens used differed in their efficacy, in order to optimise and standardise clinical practice.

Adult patients with bronchiectasis who underwent treatment aimed at P. aeruginosa eradication between January 1, 2007 and December 31, 2014 were identified from the clinical database. Historical data were collected from medical notes. P. aeruginosa eradication was considered successful if all (and at least three) bacteriologic cultures from respiratory samples collected during the 6-month period following the eradication attempt were negative for $P$. aeruginosa. Patients who remained on chronic ( $>3$ months) nebulised antibiotics were excluded from the analysis, as this treatment could suppress bacterial growth and overestimate eradication success rate. In order to select patients with recent acquisition of $P$. aeruginosa, we included only those who had never grown $P$. aeruginosa or those who were free of this bacterium for at least 2 years (and documented by five or more negative samples) before the eradication trial. All patients had a second confirmatory sample collected prior to initiation of eradication therapy.

64 patients who had at least one eradication attempt were identified. Their mean \pm SD age was $64 \pm 1.6$ years; $58 \%(\mathrm{n}=37)$ were male, and $63 \%(\mathrm{n}=39 / 62)$ of patients were receiving azithromycin $500 \mathrm{mg}$ thrice weekly. Forced expiratory volume in $1 \mathrm{~s}(\mathrm{FEV} 1)$ was $1.70 \pm 0.15 \mathrm{~L}$ and forced vital capacity was $2.89 \pm 0.19 \mathrm{~L}$.

$84 \%$ of patients $(n=54)$ received an eradication regimen that included nebulised colistimethate sodium (table 1). The most frequent regimen used was the combination of nebulised colistimethate sodium with oral ciprofloxacin, prescribed for at least 3 weeks $(n=27,42 \%)$.

Overall, the eradication success rate at 6 months was 52\% $(n=33)$, and $70 \%(n=23)$ of these patients remained $P$. aeruginosa free for at least 1 year. Treatment combinations including nebulised colistimethate sodium were more effective $(n=31 / 54,57 \%)$ than those with systemic antibiotics alone $(n=2 / 10,20 \%)$ (t-test, $\mathrm{p}=0.04$ ).

Intravenous anti-pseudomonal antibiotics $(n=9 / 18,50 \%)$ were not superior to oral ciprofloxacin $(n=21 / 35$, $60 \%$ ) in the subgroup of patients who also received nebulised antibiotics as part of their regimen (table 1). Additionally, prolonged courses of oral ciprofloxacin ( $>3$ weeks) were no more efficient than shorter treatment periods ( $\mathrm{n}=15 / 27,56 \%$ versus $\mathrm{n}=6 / 8,75 \%$ ).

@ERSpublications

Combining inhaled and systemic antibiotics appears superior for eradication of $P$. aeruginosa in bronchiectasis http://ow.ly/UpMn309KfMQ

Cite this article as: Vallières E, Tumelty K, Tunney MM, et al. Efficacy of Pseudomonas aeruginosa eradication regimens in bronchiectasis. Eur Respir J 2017; 49: 1600851 [https://doi.org/10.1183/ 13993003.00851-2016]. 
TABLE 1 Frequency and efficacy of antibiotics used as first-line eradication regimens

Nebulised colistimethate sodium (3 months) and:

No nebulised colistimethate

sodium

\begin{tabular}{lcccccccc} 
& Cipro. ( $\leqslant 3$ weeks) & Cipro. (>3 weeks) & IV (2 weeks) & Cipro.+IV & Nothing else & Cipro. & IV & Cipro.+IV \\
\hline Patients & 8 & 27 & 13 & 5 & 1 & 2 & 6 & 2 \\
Success & $6(75)$ & $15(55.5)$ & $7(54)$ & $2(40)$ & $1(100)$ & $1(17)$ & $0(0)$ & $1(50)$ \\
Total & $\mathbf{3 1 / 5 4 ( 5 7 )}$ & & & & $2 / 10(20)$ &
\end{tabular}

Data is presented as $n, \mathrm{n}(\%)$ or $\mathrm{n} / \mathrm{n}(\%)$. Cipro.: ciprofloxacin; IV: intravenous anti-pseudomonal antibiotics.
The study population was stratified according to eradication outcome (success versus failure). Demographic and clinical data of both groups were compared in order to identify factors that could have impacted eradication outcome. Age, gender, lung function and duration of infection prior to eradication were similar between groups. However, chronic azithromycin use was more frequent amongst patients who successfully cleared $P$. aeruginosa infection ( $75 \%$ versus $47 \%$, $\mathrm{p}=0.04)$.

This study suggests that eradication regimens combining nebulised and systemic antibiotics are more efficient for $P$. aeruginosa eradication than treatment without inhaled antibiotics. This finding supports results recently published by ORRIOLs et al. [4], wherein the authors randomised 35 patients who recently acquired $P$. aeruginosa infection to receive 2 weeks of intravenous antibiotics (ceftazidime and tobramycin), followed by either 3 months of nebulised tobramycin or placebo. They found that the interval for recurrence of $P$. aeruginosa infection was extended in the tobramycin group and that the treatment arm had a reduced number of exacerbations and hospitalisations during the follow-up period. However, one-third of patients in the tobramycin group experienced bronchospasm. In our cohort, respiratory symptoms associated with nebulised colistimethate sodium were infrequent as only two patients had to prematurely discontinue the inhaled treatment. Although consistent with our findings, results from the study from OrRIOLs et al. [4] must be interpreted cautiously owing to the small sample size and limitations in study design and reporting.

The potential benefit conferred by azithromycin on P. aeruginosa eradication needs further investigation. Azithromycin may contribute to biofilm disruption and may augment the action of anti-pseudomonal antibiotics [5-7].

In conclusion, this study suggests the superiority of a combination of systemic (oral or intravenous) and inhaled antibiotics as compared to systemic antibiotics alone in the initial eradication treatment of $P$. aeruginosa infection in patients with bronchiectasis. Considering the literature gap addressing $P$. aeruginosa eradication in this population, these findings should inform the design of appropriate randomised clinical trials to determine the best therapeutic approach, including the role of macrolides, in the treatment of new infections with $P$. aeruginosa in people with bronchiectasis.

Emilie Vallières ${ }^{1,3,4}$, Karen Tumelty ${ }^{4}$, Michael M. Tunney ${ }^{2,3,4}$, Rosemary Hannah ${ }^{4}$, Oonagh Hewitt ${ }^{4}$, J. Stuart Elborn ${ }^{1,3,4}$ and Damian G. Downey ${ }^{3,4}$

${ }^{1}$ School of Medicine, Dentistry and Biomedical Sciences, Queen's University Belfast, Belfast, UK. ${ }^{2}$ School of Pharmacy, Queen's University Belfast, Belfast, UK. ${ }^{3} \mathrm{CF}$ and Airways Microbiology Research Group, Queen's University Belfast, Queen's University Belfast, Belfast, UK. ${ }^{4}$ Belfast City Hospital, Belfast Health and Social Care Trust, Belfast, UK.

Correspondence: Damian G. Downey, Regional Adult Cystic Fibrosis Centre, Respiratory Research, Belfast City Hospital, Belfast, BT9 7AB, UK. E-mail: damian.downey@belfasttrust.hscni.net

Received: April 292016 | Accepted after revision: Feb 142017

Conflict of interest: Disclosures can be found alongside this article at erj.ersjournals.com

\section{References}

1 Finch S, McDonnell MJ, Abo-Leyah $\mathrm{H}$, et al. A comprehensive analysis of the impact of Pseudomonas aeruginosa colonization on prognosis in adult bronchiectasis. Ann Am Thorac Soc 2015; 12: 1602-1611.

2 Pasteur MC, Bilton D, Hill AT. British Thoracic Society guideline for non-CF bronchiectasis. Thorax 2010; 65 Suppl 1: i1-i58.

3 Valerius NH, Koch C, Høiby N. Prevention of chronic Pseudomonas aeruginosa colonisation in cystic fibrosis by early treatment. Lancet 1991; 338: 725-726.

4 Orriols R, Hernando R, Ferrer A, et al. Eradication therapy against Pseudomonas aeruginosa in non-cystic fibrosis bronchiectasis. Respiration 2015; 90: 299-305. 
5 Wagner T, Soong G, Sokol S, et al. Effects of azithromycin on clinical isolates of Pseudomonas aeruginosa from cystic fibrosis patients. Chest 2005; 128: 912-919.

6 Lutz L, Pereira DC, Paiva RM, et al. Macrolides decrease the minimal inhibitory concentration of anti-pseudomonal agents against Pseudomonas aeruginosa from cystic fibrosis patients in biofilm. BMC Microbiol 2012; 12: 196.

7 Ichimiya $\mathrm{T}$, Takeoka K, Hiramatsu $\mathrm{K}$, et al. The influence of azithromycin on the biofilm formation of Pseudomonas aeruginosa in vitro. Chemotherapy 1996; 42: 186-191.

Copyright @eERS 2017 